\title{
STABILITY AND UNIQUENESS FOR THE SPATIALLY HOMOGENEOUS BOLTZMANN EQUATION WITH LONG-RANGE INTERACTIONS
}

\author{
LAURENT DESVILLETTES, CLÉMENT MOUHOT
}

\begin{abstract}
In this paper, we prove some a priori stability estimates (in weighted Sobolev spaces) for the spatially homogeneous Boltzmann equation without angular cutoff (covering every physical collision kernels). These estimates are conditioned to some regularity estimates on the solutions, and therefore reduce the stability and uniqueness issue to the one of proving suitable regularity bounds on the solutions. We then prove such regularity bounds for a class of interactions including the so-called (non cutoff and non mollified) hard potentials and moderately soft potentials. In particular, we obtain the first result of global existence and uniqueness for these long-range interactions.
\end{abstract}

Mathematics Subject Classification (2000): 76P05 Rarefied gas flows, Boltzmann equation [See also 82B40, 82C40, 82D05].

Keywords: Boltzmann equation, spatially homogeneous, non-cutoff, long-range interactions, hard potentials, soft potentials, moderately soft potentials.

\section{Contents}

\begin{tabular}{|r|r|}
\hline 1. Introduction & 1 \\
\hline 2. Proof of the stabilitv estimates & 6 \\
\hline 3. Proof of the estimates on the propagation of smoothness & 16 \\
\hline References & 27 \\
\hline
\end{tabular}

\section{INTRODUCTION}

1.1. The Boltzmann equation. The Boltzmann equation (Cf. [5] and [6]) describes the behavior of a dilute gas when the only interactions taken into account are binary collisions. In the case when the distribution function is assumed to be independent on the position $x$, we obtain the so-called spatially homogeneous Boltzmann equation, which reads

$$
\frac{\partial f}{\partial t}(t, v)=Q(f, f)(t, v), \quad v \in \mathbb{R}^{N}, \quad t \geq 0,
$$


where $N \geq 2$ is the dimension. In equation (1.1), $Q$ is the quadratic Boltzmann collision operator, defined by the bilinear symmetrized form

$$
Q(g, f)(v)=\frac{1}{2} \int_{\mathbb{R}^{N} \times \mathbb{S}^{N-1}} B\left(\left|v-v_{*}\right|, \cos \theta\right)\left(g_{*}^{\prime} f^{\prime}+g^{\prime} f_{*}^{\prime}-g_{*} f-g f_{*}\right) d v_{*} d \sigma,
$$

where we have used the shorthands $f=f(v), f^{\prime}=f\left(v^{\prime}\right), g_{*}=g\left(v_{*}\right)$ and $g_{*}^{\prime}=g\left(v_{*}^{\prime}\right)$. Moreover, $v^{\prime}$ and $v_{*}^{\prime}$ are parametrized by

$$
v^{\prime}=\frac{v+v_{*}}{2}+\frac{\left|v-v_{*}\right|}{2} \sigma, \quad v_{*}^{\prime}=\frac{v+v_{*}}{2}-\frac{\left|v-v_{*}\right|}{2} \sigma, \quad \sigma \in \mathbb{S}^{N-1} .
$$

Finally, $\theta \in[0, \pi]$ is the deviation angle between $v^{\prime}-v_{*}^{\prime}$ and $v-v_{*}$ defined by $\cos \theta=\left(v^{\prime}-v_{*}^{\prime}\right) \cdot\left(v-v_{*}\right) /\left|v-v_{*}\right|^{2}$, and $B$ is the Boltzmann collision kernel determined by physics (related to the cross-section $\Sigma\left(v-v_{*}, \sigma\right)$ by the formula $B=\left|v-v_{*}\right| \Sigma$ ). We also formally denote

$$
Q^{+}(f, f)(v)=\int_{\mathbb{R}^{N} \times \mathbb{S}^{N-1}} B\left(\left|v-v_{*}\right|, \cos \theta\right) f_{*}^{\prime} f^{\prime} d v_{*} d \sigma
$$

the gain part of $Q$, and

$$
L(f)(v)=\int_{\mathbb{R}^{N} \times \mathbb{S}^{N-1}} B\left(\left|v-v_{*}\right|, \cos \theta\right) f_{*} d v_{*} d \sigma
$$

the linear operator appearing in the loss part $Q^{-}$of $Q$.

Boltzmann's collision operator has the fundamental properties of conserving mass, momentum and energy

$$
\int_{\mathbb{R}^{N}} Q(f, f) \phi(v) d v=0, \quad \phi(v)=1, v,|v|^{2},
$$

and satisfying Boltzmann's $H$ theorem, which writes (at the formal level)

$$
-\frac{d}{d t} \int_{\mathbb{R}^{N}} f \log f d v=-\int_{\mathbb{R}^{N}} Q(f, f) \log (f) d v \geq 0 .
$$

1.2. Assumptions on the collision kernel. We shall consider the following assumptions on the collision kernel $B$ :

H1. It takes the following tensorial form (with $\Phi, b$ nonnegative functions)

$$
B\left(\left|v-v_{*}\right|, \cos \theta\right)=\Phi\left(\left|v-v_{*}\right|\right) b(\cos \theta) .
$$

H2. The angular part is nonnegative, smooth (or at least locally integrable) for $\theta \in(0, \pi]$, and such that

$$
b(\cos \theta) \sim_{\theta \rightarrow 0} C_{b} \theta^{-(N-1)-\nu}
$$

with $\nu \in(-\infty, 2)$ and $C_{b}>0$. 
As for the "kinetic" part $\Phi$, we make one of the following assumptions:

H3-1. The function $z \mapsto \Phi(|z|)$ is strictly positive, $C^{\infty}$, such that

$$
\Phi(|z|) \sim_{|z| \rightarrow+\infty} C_{\Phi}|z|^{\gamma},
$$

for some $C_{\Phi}>0$ and $\gamma \in(0,1]$, and satisfies the bounds

$$
\forall z \in \mathbb{R}^{N}, p \in \mathbb{N}^{*}, \quad\left|\Phi^{(p)}(|z|)\right| \leq C_{\Phi, p},
$$

for some $C_{\Phi, p}>0$.

H3-2. The function $z \mapsto \Phi(|z|)$ is strictly positive, $C^{\infty}$, such that

$$
\Phi(|z|) \sim_{|z| \rightarrow+\infty} C_{\Phi}|z|^{\gamma}
$$

for some $C_{\Phi}>0$ and $\gamma \in(-N, 0]$, and satisfies the bounds

$$
\forall z \in \mathbb{R}^{N}, p \in \mathbb{N}^{*}, \quad\left|\Phi^{(p)}(|z|)\right| \leq C_{\Phi, p},
$$

for some $C_{\Phi, p}>0$.

H3-3. The function $z \mapsto \Phi(|z|)$ is given by the explicit formula

$$
\Phi(|z|)=C_{\Phi}|z|^{\gamma}
$$

for some $C_{\Phi}>0$ and $\gamma \in(0,1]$.

H3-4. The function $z \mapsto \Phi(|z|)$ is given by the explicit formula

$$
\Phi(|z|)=C_{\Phi}|z|^{\gamma}
$$

for some $C_{\Phi}>0$ and $\gamma \in(-N, 0]$.

Our assumptions (more precisely, H3-3) cover in dimension 3 the hard spheres collision kernel $B\left(\left|v-v_{*}\right|, \cos \theta\right)=$ cst $\left|v-v_{*}\right|$. It also covers (still in dimension 3) collision kernels deriving from interaction potentials behaving like inverse-power laws. More precisely for an interaction potential $V(r)=\operatorname{cst} r^{-s}, B$ satisfies our assumptions with the formulas $\gamma=(s-4) / s$ and $\nu=2 / s$ (see [5]). One traditionally calls hard potentials the case $s>4$ (for which $0<\gamma<1$, and which corresponds to H3-3), Maxwell molecules the case $s=4$ (which corresponds to H3-2 with $\gamma=0$ ), and soft potentials the case $1<s<4$ (for which $-N<\gamma<0$, and which corresponds to H3-4).

Assumptions H3-1 and H3-2 correspond to cases when $B$ is artificially smoothed around 0 with respect to $v-v_{*}$.

Since $\nu=2 / s$ for potentials in $r^{-s}$, only the non-negative $\nu$ are physically meaningful (as far as inverse power laws are concerned). The case of negative $\nu$, corresponding to the so-called angular cutoff, is a simplification. 
1.3. Goals, existing results and difficulties. The stability of the spatially homogeneous Boltzmann equation for hard potentials (or hard spheres, or Maxwellian molecules) with angular cutoff was proven, in weighted $L^{1}$ spaces, by Arkeryd [2]. The special structure of the Maxwellian molecules makes it possible to prove the stability (and consequently the uniqueness) of the corresponding spatially homogeneous Boltzmann equation without angular cutoff using Fourier transform and Wasserstein-like distances (Cf. 24]). A recent work of Fournier 13 shows by probabilistic means that this stability also holds without angular cutoff for "kinetic" sections $\Phi$ which are not of Maxwellian molecules type but are bounded and smooth (this more or less corresponds to our hypothesis H3-2), and for moderate angular singularities (that is $\nu \in] 0,1[$ ). This recent paper is an important step in the application of contraction metrics approach for non constant collision kernels, even if up to now it does not consider physical collision kernels apart from the Maxwell molecules one.

Hence, as far as we know, no stability (or uniqueness) result is known for "true" hard or soft potentials. Here, we show that stability holds for any kind of interactions, as soon as suitable regularity bounds are at hand. Then, we prove these required regularity bounds for a class of models including "true" hard potentials and moderately soft potentials. Our approach is complementary to the one of Fournier 13 in the sense that our stability is in a stronger space (that is in a weighted $W^{1,1}$ space instead of a measure space), for more general cross-sections, but leads to a uniqueness result holding for a smaller set of initial data. Our method of proof is also completely different. It is based on the use of integrations by parts for finite differences of a special kind.

We shall devote a separate forthcoming work [10] to the question of the asymptotic behavior when $t \rightarrow+\infty$ of the solution of the spatially homogeneous Boltzmann equation without cutoff, on the basis of the Cauchy theorems established in the present work, new entropy production estimates, and the approach developed in [9].

1.4. Notation. We denote $\langle\cdot\rangle=\left(1+|\cdot|^{2}\right)^{1 / 2}$. We shall systematically use the following notations $(s \in \mathbb{R}, p \in[1,+\infty), k \in \mathbb{N})$

$$
\|f\|_{L_{s}^{p}}^{p}:=\int_{\mathbb{R}^{N}}|f(v)|^{p}\langle v\rangle^{p s} d v, \quad\|f\|_{L_{s}^{\infty}}:=\sup _{v \in \mathbb{R}^{N}}|f(v)|\langle v\rangle^{s}
$$

and

$$
\|f\|_{W_{s}^{k, p}}^{p}:=\sum_{0 \leq|i| \leq k}\left\|\partial^{i} f\right\|_{L_{s}^{p}}^{p}, \quad\|f\|_{W_{s}^{k, \infty}}:=\sum_{0 \leq|i| \leq k}\left\|\partial^{i} f\right\|_{L_{s}^{\infty}},
$$

where $\partial^{i}$ denotes the partial derivative related to the multi-index $i$. In the case $p=2$, we denote $H_{s}^{k}=W_{s}^{k, 2}$. We finally use the notation $x_{+}$for the nonnegative part of $x \in \mathbb{R}$, defined by $x_{+}=\max \{x, 0\}$. 
1.5. Statement of the results. We first state the key a priori stability theorem for moderate angular singularities:

Theorem 1.1. Let $B$ be a collision kernel which satisfies $\mathbf{H 1 - H 2}$ with $\nu<1$, and let $f, g \in L^{\infty}\left([0, T] ; L_{2}^{1} \cap L \log L\left(\mathbb{R}^{N}\right)\right)$ be two nonnegative solutions to the spatially homogeneous Boltzmann equation associated to $B$, on some time interval $[0, T]$.

We assume first that $B$ satisfies $\mathbf{H 3 - 1 , ~ H 3 - 2}$ or $\mathbf{H 3 - 3 . ~ F o r ~ a n y ~} q \geq 2$, we have the following a priori bound

$$
\forall t \in[0, T], \quad\|f(t, \cdot)-g(t, \cdot)\|_{L_{q}^{1}} \leq\left\|f_{0}-g_{0}\right\|_{L_{q}^{1}} \exp \left(C_{s} t\right),
$$

with

$$
C_{s}=\operatorname{cst}\left(\sup _{t \in[0, T]}\|f(t, \cdot)\|_{W_{q+(1+\gamma)_{+}}^{1,1}}+\sup _{t \in[0, T]}\|g(t, \cdot)\|_{W_{q+(1+\gamma)_{+}}^{1,1}}\right) .
$$

We assume then that $B$ satisfies $\mathbf{H 3 - 4}$. We still have (1.3) for any $q \geq 2$, but with

$$
C_{s}=\operatorname{cst}\left(\sup _{t \in[0, T]}\|f(t, \cdot)\|_{W_{q+(1+\gamma)_{+}}^{1,1} \cap L^{p}}+\sup _{t \in[0, T]}\|g(t, \cdot)\|_{W_{q+(1+\gamma)_{+}}^{1,1} \cap L^{p}}\right),
$$

where $p>N /(N+\gamma)$ if $\gamma+1 \geq 0$, and else

$$
\begin{aligned}
C_{s} & =\operatorname{cst}\left(\sup _{t \in[0, T]}\|f(t, \cdot)\|_{W_{q+(1+\gamma)_{+}}^{1,1} \cap L^{p_{1}}}+\sup _{t \in[0, T]}\|\nabla f(t, \cdot)\|_{L^{p_{2}}}\right. \\
& \left.+\sup _{t \in[0, T]}\|g(t, \cdot)\|_{W_{q+(1+\gamma)_{+}}^{1,1} \cap L^{p_{1}}}+\sup _{t \in[0, T]}\|\nabla g(t, \cdot)\|_{L^{p_{2}}}\right),
\end{aligned}
$$

where $p_{1}>N /(N+\gamma)$ and $p_{2}>N /(N+\gamma+1)$.

We also give a proposition stating the stability result for strong angular singularities. For the sake of simplicity, we do not write down the explicit estimate in this case for "true" soft potentials (case H3-4), but it can be obtained from our proof.

Proposition 1.2. Let $B$ be a collision kernel which satisfies $\mathbf{H 1 - H 2}$ with $1 \leq \nu<$ 2 , and let $0 \leq f(t, \cdot), g(t, \cdot) \in L_{2}^{1}$ be two solutions to the spatially homogeneous Boltzmann equation (1.1) associated to $B$, on some time interval $[0, T]$. We assume first that $B$ satisfies $\mathbf{H 3 - 1 , ~ H 3 - 2 ~ o r ~} \mathbf{H 3 - 3 . ~ F o r ~ a n y ~} q \geq 4$, estimate (1.3) holds with

$$
C_{s}=\operatorname{cst}\left(\sup _{t \in[0, T]}\|f(t, \cdot)\|_{W_{q+(2+\gamma)_{+}}^{2,1}}+\sup _{t \in[0, T]}\|g(t, \cdot)\|_{W_{q+(2+\gamma)_{+}}^{2,1}}\right) .
$$


Second, we state a theorem summing up what we obtain for the Cauchy theory by combining the previous a priori stability estimates (that is, Theorem 1.1) with results on the propagation of smoothness which are either already known (when $\nu<0$, that is, for cutoff cross-sections) or new (when $\nu \in[0,1)$, that is for non cutoff cross-sections).

Theorem 1.3. Let $B$ be a collision kernel which satisfies $\mathbf{H 1 - H 2}$ with $\nu<1$.

- If $B$ satisfies $\mathbf{H 3 - 1 , ~ H 3 - 2 ~ o r ~ H 3 - 3 ~ a n d ~} f(0, \cdot)$ is an initial datum belonging to $W_{q}^{1,1}$ for some $q \geq 2$, there is a unique global solution to eq. (1.1) in the space $W_{q}^{1,1}$.

- If $B$ satisfies H3-4 for $\gamma \geq-1$, and $f(0, \cdot)$ is an initial datum belonging to $W_{q}^{1,1} \cap L^{p}$ for $p>\frac{N}{N+\gamma}$ and $q \geq 2$, there is a unique local (that is, on a certain time interval $[0, T])$ solution in the space $W_{q}^{1,1} \cap L^{p}$. Moreover, this solution is global (that is, $T=+\infty$ ) when $\gamma \in(-\nu, 0]$ and $q$ is big enough (depending on $\gamma, \nu$ ).

Remark 1.4. Hence in dimension $N=3$ where $\nu=2 / s$ and $\gamma=(s-4) / s$ for potentials in $r^{-s}$, this theorem yields global existence and uniqueness results for any "true" hard potentials $(4<s<+\infty)$ and "true" moderately soft potentials $(2<s<4)$. Indeed in these cases, one has $0<\nu<1$ and $-\nu<\gamma \leq 1$. Note however that our method, like that of Fournier, does not seem to work for strong angular singularities $(1<\nu<2)$, even if the kinetic part $\Phi$ of the cross-section is very smooth. As a consequence, Proposition 1.2 has not yet found an application (that is, it gives a result of uniqueness without existence...)

1.6. Plan of the paper. Section 2 is devoted to the proof of Theorem [1.1 and Proposition 1.2. The case of "true" soft potentials H3-4 is somewhat different from the others, and it is therefore treated separately.

Then, Theorem 1.3 is proven in section 3. Once again, the case of "true" soft potentials deserves a special treatment.

\section{Proof of the Stability estimates}

Proof of Theorem 1.1 and Proposition 1.2, Without restriction (since $Q$ is taken in symmetrized form), we replace in the whole paper the cross-section $B$ by its symmetrized form with support included in $\theta \in[0, \pi / 2]$ :

$$
B_{\mathrm{sym}}\left(\left|v-v_{*}\right|, \cos \theta\right)=\left[B\left(\left|v-v_{*}\right|, \cos \theta\right)+B\left(\left|v-v_{*}\right|, \cos (\pi-\theta)\right)\right] \mathbf{1}_{\cos \theta \geq 0}
$$

where $\mathbf{1}_{E}$ denotes the usual characteristic function of the set $E$.

Let $B$ be a collision kernel which satisfies $\mathbf{H 1 - H 2}$ and one of the assumptions H3, and $f(t, \cdot), g(t, \cdot) \geq 0$ be two solutions on $[0, T]$ to the associated spatially 
STABILITY FOR THE BOLTZMANN EQUATION WITH LONG-RANGE INTERACTIONS 7

homogeneous Boltzmann equation (1.1). Let us define $D=f-g$ and $S=f+g$. The evolution equation for $D$ reads

$$
\frac{\partial D}{\partial t}=Q(f, f)-Q(g, g)=Q(S, D)
$$

2.1. Hard potentials or mollified soft potentials. Let us first assume H3-1, H3-2 or H3-3.

We split $b=b_{c}^{\varepsilon}+b_{r}^{\varepsilon}$ with $b_{c}^{\varepsilon}=b \mathbf{1}_{\theta \in[\varepsilon, \pi / 2]}$ (the cutoff part), $b_{r}^{\varepsilon}=1-b_{c}^{\varepsilon}$ (the remaining part), and $\varepsilon \in(0, \pi / 2]$ to be fixed later. This induces corresponding splittings of the collision kernel $B=B_{c}+B_{r}$ and of the collision operator $Q=Q_{c}+Q_{r}$.

Then, we have

$$
\frac{d}{d t}\|D\|_{L_{q}^{1}} \leq \int_{\mathbb{R}^{N}} Q_{r}(S, D) \operatorname{sgn}(D)\langle v\rangle^{q} d v+\int_{\mathbb{R}^{N}} Q_{c}(S, D) \operatorname{sgn}(D)\langle v\rangle^{q} d v=: I_{1}+I_{2} .
$$

For the cutoff part, we estimate

$$
\begin{aligned}
& 2 I_{2}=2 \int_{\mathbb{R}^{N}} Q_{c}(S, D) \operatorname{sgn}(D)\langle v\rangle^{q} d v \\
&=\int_{v, v_{*}, \sigma}\left[S_{*}^{\prime} D^{\prime}+S^{\prime} D_{*}^{\prime}-S_{*} D-S D_{*}\right] \operatorname{sgn}(D)\langle v\rangle^{q} B_{c} \\
& \leq \int_{v, v_{*}, \sigma}\left(S_{*}^{\prime}\left|D^{\prime}\right|+S^{\prime}\left|D^{\prime}\right|_{*}\right.\left.-S_{*}|D|-S\left|D_{*}\right|\right)\langle v\rangle^{q} B_{c}+2 \int_{v, v_{*}, \sigma} S\left|D_{*}\right|\langle v\rangle^{q} B_{c} \\
&=2 \int_{\mathbb{R}^{N}} Q_{c}(S,|D|)\langle v\rangle^{q} d v+2 \int_{v, v_{*}, \sigma} S\left|D_{*}\right|\langle v\rangle^{q} B_{c},
\end{aligned}
$$

which implies

$$
\begin{array}{rl}
I_{2} \leq C \int_{\mathbb{R}^{N}} Q_{c}(S,|D|)\langle v\rangle^{q} & d v \\
& +C_{\varepsilon} \int_{\mathbb{R}^{N} \times \mathbb{R}^{N}}\left|D_{*}\right| S\langle v\rangle^{q+\gamma_{+}}\left\langle v_{*}\right\rangle^{\gamma_{+}} d v_{*} d v=: I_{2,1}+I_{2,2},
\end{array}
$$

where the constant $C_{\varepsilon}>0$ depends on $\varepsilon>0$ via the $L^{1}$ norm of $b_{c}^{\varepsilon}$ on the sphere $\mathbb{S}^{N-1}$ (which possibly blows up as $\varepsilon \rightarrow 0$ ).

The $I_{2,2}$ term is controlled (for $q \geq \gamma_{+}$) by

$$
I_{2,2} \leq C_{\varepsilon} C_{q}\|D\|_{L_{\gamma_{+}}^{1}} \leq C_{\varepsilon}^{\prime}\|D\|_{L_{q}^{1}}
$$

The $I_{2,1}$ term writes, using the pre-post-collisional change of variable (see [27, Chapter 1, Section 4.5]):

$$
I_{2,1}=\int_{\mathbb{R}^{N} \times \mathbb{R}^{N}}\left|D_{*}\right| S \Phi\left(\left|v-v_{*}\right|\right)\left(\int_{\mathbb{S}^{N-1}}\left[\left\langle v^{\prime}\right\rangle^{q}+\left\langle v_{*}^{\prime}\right\rangle^{q}-\langle v\rangle^{q}-\left\langle v_{*}\right\rangle^{q}\right] b_{c}^{\varepsilon} d \sigma\right) d v d v_{*} .
$$


Then, we shall prove (for $q>2$ )

$$
\begin{gathered}
\left(\int_{\mathbb{S}^{N-1}}\left[\left\langle v^{\prime}\right\rangle^{q}+\left\langle v_{*}^{\prime}\right\rangle^{q}-\langle v\rangle^{q}-\left\langle v_{*}\right\rangle^{q}\right] b_{c}^{\varepsilon} d \sigma\right) \\
\leq \operatorname{cst}\left(\int_{\mathbb{S}^{N-1}}\left[\left|v^{\prime}\right|^{q}+\left|v_{*}^{\prime}\right|^{q}-|v|^{q}-\left|v_{*}\right|^{q}\right] b_{c}^{\varepsilon} d \sigma\right) \\
\leq C_{\varepsilon}\left[|v|^{q-1}\left|v_{*}\right|+|v|\left|v_{*}\right|^{q-1}\right]-K\left[|v|^{q}+\left|v_{*}\right|^{q}\right]
\end{gathered}
$$

for again some constant $C_{\varepsilon}>0$ possibly blowing-up as $\varepsilon \rightarrow 0$, and some constant $K>0$ independent of $\varepsilon$.

The proof of this inequality is straightforward, using the kind of Povzner inequalities in [29, 18. Indeed, [18, Lemma 1] implies that

$$
\begin{aligned}
& \left\langle v^{\prime}\right\rangle^{q}+\left\langle v_{*}^{\prime}\right\rangle^{q}-\langle v\rangle^{q}-\left\langle v_{*}\right\rangle^{q} \\
& \quad \leq 2^{q+1}\left[\langle v\rangle^{q-1}\left\langle v_{*}\right\rangle+\left\langle v_{*}\right\rangle^{q-1}\langle v\rangle\right] \cos \theta \sin \theta-K_{q}\left[\langle v\rangle^{q}+\left\langle v_{*}\right\rangle^{q}\right] \cos ^{2} \theta \sin ^{2} \theta
\end{aligned}
$$

for some constant $K_{q}>0$ depending only on $q$ (note that the proof in [18] is done in dimension 3 but straightforwardly extends to any dimension). Note also that in the case of moderate angular singularities $\nu<1$, the constant $C_{\varepsilon}$ indeed does not blow up as $\varepsilon$ goes to infinity.

Hence, using that $q \geq 1+\gamma_{+}$, that $q-1+\gamma_{+} \leq q$ (since $\gamma_{+} \leq 1$ and $q>2$ ), and also that

$$
\int_{\mathbb{R}^{N}} S\left(v_{*}\right) \Phi\left(v-v_{*}\right) d v_{*} \geq \operatorname{cst}\langle v\rangle^{\gamma}
$$

thanks to the entropy bounds on $f$ and $g$, we get

$$
I_{2,1} \leq C_{\varepsilon}^{\prime} C_{q}\|D\|_{L_{q}^{1}}-K^{\prime}\|D\|_{L_{q+\gamma}^{1}}
$$

with $C_{\varepsilon}^{\prime}$ possibly blowing up as $\varepsilon \rightarrow 0$, and $K^{\prime}$ independent of $\varepsilon$.

The remaining non cutoff part writes

$$
\begin{aligned}
\int_{\mathbb{R}^{N}} Q_{r}(S, D) & \operatorname{sgn}(D)\langle v\rangle^{q} d v \\
= & \int_{\mathbb{R}^{N} \times \mathbb{R}^{N} \times \mathbb{S}^{N-1}} \Phi b_{r}\left[S_{*}^{\prime} D^{\prime}-S_{*} D\right] \operatorname{sgn}(D)\langle v\rangle^{q} d v d v_{*} d \sigma \\
+ & \int_{\mathbb{R}^{N} \times \mathbb{R}^{N} \times \mathbb{S}^{N-1}} \Phi b_{r}\left[D_{*}^{\prime} S^{\prime}-D_{*} S\right] \operatorname{sgn}(D)\langle v\rangle^{q} d v d v_{*} d \sigma=: I_{1,1}+I_{1,2} .
\end{aligned}
$$


The $I_{1,1}$ term is the easiest to deal with:

$$
\begin{gathered}
I_{1,1}=\int_{v, v_{*}, \sigma}\left[S_{*}^{\prime} D^{\prime}-S_{*} D\right] \operatorname{sgn}(D)\langle v\rangle^{q} \Phi\left(\left|v-v_{*}\right|\right) b_{r}^{\varepsilon} d v d v_{*} d \sigma \\
=\int_{v, v_{*}, \sigma} S_{*}\left[D \operatorname{sgn}\left(D^{\prime}\right)\left\langle v^{\prime}\right\rangle^{q}-|D|\langle v\rangle^{q}\right] \Phi\left(\left|v-v_{*}\right|\right) b_{r}^{\varepsilon} d v d v_{*} d \sigma \\
\leq \int_{v, v_{*}, \sigma} S_{*}|D|\left[\left\langle v^{\prime}\right\rangle^{q}-\langle v\rangle^{q}\right] \Phi\left(\left|v-v_{*}\right|\right) b_{r}^{\varepsilon} d v d v_{*} d \sigma \\
\leq \int_{v, v_{*}} S_{*}|D| \Phi\left(\left|v-v_{*}\right|\right)\left|\int_{\mathbb{S}^{N-1}}\left[\left\langle v^{\prime}\right\rangle^{q}-\langle v\rangle^{q}\right] b_{r}^{\varepsilon} d \sigma\right| d v d v_{*} .
\end{gathered}
$$

Then we shall prove a simple lemma, which is a variant of [8, Lemma 2.3].

Lemma 2.1. Let $q \geq 2$, then

$$
\begin{aligned}
& \mid \int_{\mathbb{S}^{N-1}}\left[\left\langle v^{\prime}\right\rangle^{q}-\langle v\rangle^{q}\right] b(\cos \theta) d \sigma \mid \\
& \leq C\left(\int_{\mathbb{S}^{N-1}} b(\cos \theta) \sin \theta / 2 d \sigma\right)\left|v-v_{*}\right|\left[\langle v\rangle^{q-1}+\left\langle v_{*}\right\rangle^{q-1}\right] .
\end{aligned}
$$

Let $q \geq 4$, then

$$
\begin{aligned}
& \left|\int_{\mathbb{S}^{N-1}}\left[\left\langle v^{\prime}\right\rangle^{q}-\langle v\rangle^{q}\right] b(\cos \theta) d \sigma\right| \\
& \leq C\left(\int_{\mathbb{S}^{N-1}} b(\cos \theta)(\sin \theta / 2)^{2} d \sigma\right)\left|v-v_{*}\right|^{2}\left[\langle v\rangle^{q-2}+\left\langle v_{*}\right\rangle^{q-2}\right] .
\end{aligned}
$$

In those formulas, the constants $C>0$ depend only on $q$. The same formulas are true when $\left(v, v^{\prime}\right)$ is replaced by $\left(v_{*}, v_{*}^{\prime}\right)$.

Proof of Lemma 2.1. The proof is straightforward by using integral Taylor expansions of $u \in[0,1] \mapsto\left\langle v_{u}^{\prime}\right\rangle^{q} \in \mathbb{R}$ (denoting $\left.v_{u}^{\prime}=(1-u) v+u v^{\prime}\right)$.

At first order, one gets

$$
\left\langle v^{\prime}\right\rangle^{q}-\langle v\rangle^{q}=q \int_{0}^{1}\left\langle v_{u}^{\prime}\right\rangle^{q-2} v_{u}^{\prime} \cdot\left(v^{\prime}-v\right) d u,
$$

which is enough to prove the first inequality.

At second order, one gets

$$
\begin{aligned}
\left\langle v^{\prime}\right\rangle^{q}-\langle v\rangle^{q} & =q\langle v\rangle^{q-2} v \cdot\left(v^{\prime}-v\right) \\
& +q \int_{0}^{1}\left[\left\langle v_{u}^{\prime}\right\rangle^{q-2}\left|v^{\prime}-v\right|^{2}+(q-2)\left\langle v_{u}^{\prime}\right\rangle^{q-4}\left|v_{u}^{\prime}\right|^{2}\left|v^{\prime}-v\right|^{2}\right](1-u) d u .
\end{aligned}
$$


This is enough to prove the second inequality as soon as one notices that

$$
\int_{\mathbb{S}^{N-1}}\langle v\rangle^{q-2} v \cdot\left(v^{\prime}-v\right) b(\cos \theta) d \sigma=0
$$

by gathering antipodal points of the $(N-2)$-dimensional sphere $\mathbb{S}^{N-1} \cap\left(v-v_{*}\right)^{\perp}$.

We turn back to the proof of Theorem 1.1 and Proposition 1.2 in the case of hard or smoothed soft potentials.

From Lemma 2.1, we deduce that when $q \geq 2$,

$$
\left|\int_{\mathbb{S}^{N-1}}\left[\left\langle v^{\prime}\right\rangle^{q}-\langle v\rangle^{q}\right] b_{r}^{\varepsilon} d \sigma\right| \leq C m_{1}\left(b_{r}^{\varepsilon}\right)\langle v\rangle^{q}\left\langle v_{*}\right\rangle^{q},
$$

where $C>0$ is independent of $\varepsilon$, and

$$
m_{1}\left(b_{r}^{\varepsilon}\right)=\int_{\mathbb{S}^{N-1}} b_{r}^{\varepsilon}(\cos \theta) \sin \theta / 2 d \sigma
$$

is a finite quantity which goes to 0 as $\varepsilon$ goes to 0 , under the assumption $\nu \in(0,1)$. For $q \geq 4$, one also has the control

$$
\left|\int_{\mathbb{S}^{N-1}}\left[\left\langle v^{\prime}\right\rangle^{q}-\langle v\rangle^{q}\right] b_{r}^{\varepsilon} d \sigma\right| \leq C m_{2}\left(b_{r}^{\varepsilon}\right)\langle v\rangle^{q}\left\langle v_{*}\right\rangle^{q}
$$

with

$$
m_{2}\left(b_{r}^{\varepsilon}\right)=\int_{\mathbb{S}^{N-1}} b_{r}^{\varepsilon}(\cos \theta)(\sin \theta / 2)^{2} d \sigma
$$

which is finite and goes to 0 as $\varepsilon$ goes to 0 , for any $\nu<2$ (that is the whole physical range).

Therefore assuming $q \geq 2$ when $\nu<1$ (case of Theorem 1.1) or $q \geq 4$ when $1 \leq \nu<2$ (case of Proposition 1.2), we obtain that $I_{1,1}$ is controlled by $\|D\|_{L_{q+\gamma_{+}}^{1}}$ times some constant which goes to 0 as $\varepsilon$ goes to 0 . Thus

$$
I_{1,1} \leq \frac{K^{\prime}}{4}\|D\|_{L_{q+\gamma_{+}}^{1}}
$$

for $\varepsilon$ small enough.

We now come to the most difficult term to estimate, and the crucial point in the proof. That is the use of suitable changes of variables which play (loosely speaking) the role of some integration by parts for the "integral differentiation-like operators" appearing in the collision operator for grazing collisions.

At this point, in order to keep tractable notations, we keep on with the proof only under assumption H3-3. It can be checked easily that the proof also works under assumptions H3-1 and H3-2 (replacing $\gamma$ by $\gamma_{+}$if necessary, and using bounds on the derivatives of $\Phi)$. 
The $I_{1,2}$ term writes

$$
\begin{aligned}
& I_{1,2}=\int_{v, v_{*}, \sigma}\left[D_{*}^{\prime} S^{\prime}-D_{*} S\right] \operatorname{sgn}(D)\langle v\rangle^{q}\left|v-v_{*}\right|^{\gamma} b_{r}^{\varepsilon} d v d v_{*} d \sigma \\
& =\int_{v, v_{*}, \sigma} D_{*} S\left[\operatorname{sgn}\left(D^{\prime}\right)\left\langle v^{\prime}\right\rangle^{q}-\operatorname{sgn}(D)\langle v\rangle^{q}\right]\left|v-v_{*}\right|^{\gamma} b_{r}^{\varepsilon} d v d v_{*} d \sigma \\
& \leq \int_{v, v_{*}, \sigma} D_{*} \operatorname{sgn}(D)\langle v\rangle^{q}\left(\frac{S\left(\phi_{\sigma}\left(v, v_{*}\right)\right)}{(\cos \theta / 2)^{N+\gamma}}-S\right)\left|v-v_{*}\right|^{\gamma} b_{r}^{\varepsilon} d v d v_{*} d \sigma \\
& \leq \int_{v, v_{*}}\left|D_{*}\right|\langle v\rangle^{q}\left|\int_{\mathbb{S}^{N-1}}\left(\frac{S\left(\phi_{\sigma}\left(v, v_{*}\right)\right)}{(\cos \theta / 2)^{N+\gamma}}-S\right) b_{r}^{\varepsilon} d \sigma\right|\left|v-v_{*}\right|^{\gamma} d v d v_{*},
\end{aligned}
$$

where we have used the change of variable from cancellation lemmas in [1] (which is possible since $b$ has its support included in $[0, \pi / 2])$. The variable $\phi_{\sigma}\left(v, v_{*}\right)$ denotes the inverse application of $v \mapsto v^{\prime}$ keeping $v_{*}$ and $\sigma$ frozen (it is given explicitly in [1]). Let us denote $\bar{v}=\phi_{\sigma}\left(v, v_{*}\right)$.

We split the integral on the sphere into three parts

$$
\begin{aligned}
& \langle v\rangle^{q} \int_{\mathbb{S}^{N-1}}\left(\frac{S(\bar{v})}{(\cos \theta / 2)^{N+\gamma}}-S\right) b_{r}^{\varepsilon} d \sigma \\
& =\int_{\mathbb{S}^{N-1}} \frac{S(\bar{v})\langle\bar{v}\rangle^{q}-S(v)\langle v\rangle^{q}}{(\cos \theta / 2)^{N+\gamma}} b_{r}^{\varepsilon} d \sigma \\
& +\int_{\mathbb{S}^{N-1}}\left(\frac{1}{(\cos \theta / 2)^{N+\gamma}}-1\right) b_{r}^{\varepsilon} d \sigma S(v)\langle v\rangle^{q}+\int_{\mathbb{S}^{N-1}}\left(\frac{\langle v\rangle^{q}-\langle\bar{v}\rangle^{q}}{(\cos \theta / 2)^{N+\gamma}}\right) S(\bar{v}) b_{r}^{\varepsilon} d \sigma,
\end{aligned}
$$

which yields a corresponding splitting of $I_{1,2}$ into three parts $I_{1,2,1}+I_{1,2,2}+I_{1,2,3}$.

For the $I_{1,2,3}$ term, we use again the change of variable defined above, but backward:

$$
\left|I_{1,2,3}\right| \leq C \int_{v, v_{*}}\left|D_{*}\right| S(v)\left|v-v_{*}\right|^{\gamma}\left(\int_{\mathbb{S}^{N-1}}\left|\left\langle v^{\prime}\right\rangle^{q}-\langle v\rangle^{q}\right| b_{r}^{\varepsilon} d \sigma\right) d v d v_{*},
$$

and we apply again Lemma 2.1 (with $q \geq 2$ if $\nu<1$ or $q \geq 4$ if $1 \leq \nu<2$ ) to get by choosing $\varepsilon$ small enough

$$
I_{1,2,3} \leq \frac{K^{\prime}}{4}\|D\|_{L_{q+\gamma}^{1}} .
$$

For the $I_{1,2,2}$ term, we have

$$
\left|\frac{1}{(\cos \theta / 2)^{N+\gamma}}-1\right| \leq C(1-\cos \theta),
$$

and so

$$
I_{1,2,2} \leq C C_{s} m_{2}\left(b_{r}^{\varepsilon}\right)\|D\|_{L_{q+\gamma}^{1}} .
$$


Hence, choosing again $\varepsilon$ small enough, we get

$$
I_{1,2,2} \leq \frac{K^{\prime}}{4}\|D\|_{L_{q+\gamma}^{1}}
$$

Finally, for the $I_{1,2,1}$ term, we denote $\bar{v}_{u}=(1-u) v+u \bar{v}$ for $u \in[0,1]$ and we Taylor-expand $v \mapsto S(v)\langle v\rangle^{q}$. Let us first suppose that $\nu<1$. Then, it is enough to go to first order:

$$
S(\bar{v})\langle\bar{v}\rangle^{q}-S(v)\langle v\rangle^{q}=\int_{0}^{1} \nabla\left(S\langle\cdot\rangle^{q}\right)\left(\bar{v}_{u}\right) \cdot(\bar{v}-v) d u,
$$

and using the identity $|\bar{v}-v|=\tan \theta / 2\left|v-v_{*}\right|$, we get

$$
I_{1,2,1} \leq C \int_{\mathbb{R}^{2 N} \times \mathbb{S}^{N-1} \times[0,1]}\left|\nabla\left(S\langle\cdot\rangle^{q}\right)\right|\left(\bar{v}_{u}\right)\left|v-v_{*}\right|^{\gamma+1}(\tan \theta / 2) b_{r}^{\varepsilon}\left|D_{*}\right| d v d v_{*} d \sigma d u .
$$

When $u, v_{*}$ and $\sigma$ are fixed, the change of variable $v \rightarrow \bar{v}_{u}$ has its Jacobian determinant bounded by a constant, and for any $u \in[0,1]$,

$$
\left|v-v_{*}\right|^{\gamma+1} \leq C\left|\bar{v}_{u}-v_{*}\right|^{\gamma+1},
$$

hence

$$
\begin{array}{r}
I_{1,2,1} \leq C \int_{[0,1]} \int_{\mathbb{R}^{2 N} \times \mathbb{S}^{N-1}}\left|\nabla\left(S\langle\cdot\rangle^{q}\right)(v)\right|\left|v-v_{*}\right|^{\gamma+1}(\tan \theta / 2) b_{r}^{\varepsilon}\left|D_{*}\right| d v d v_{*} d \sigma d u \\
\leq C\left(\int_{\mathbb{S}^{N-1}}(\tan \theta / 2) b_{r}^{\varepsilon} d \sigma\right)\|S\|_{W_{q+\gamma+1}^{1,1}}\|D\|_{L_{\gamma+1}^{1}},
\end{array}
$$

for some constant $C>0$ independent of $\varepsilon$.

Thus, remembering that $\nu<1$, we have

$$
\int_{\mathbb{S}^{N-1}}(\sin \theta / 2) b_{r}^{\varepsilon} d \sigma<+\infty
$$

and so (for $q \geq \gamma+1$ )

$$
I_{1,2,1} \leq C C_{s}\|D\|_{L_{q}^{1}}
$$

Let us now briefly explain how to adapt this proof when $1 \leq \nu<2$ (case of Proposition 1.2). In order to cancel singularities of order 2, we Taylor-expand at second order:

$S(\bar{v})\langle\bar{v}\rangle^{q}-S(v)\langle v\rangle^{q}=\nabla\left(S\langle\cdot\rangle^{q}\right)(v) \cdot(\bar{v}-v)+\int_{0}^{1} \nabla^{2}\left(S\langle\cdot\rangle^{q}\right)\left(\bar{v}_{u}\right) \cdot(\bar{v}-v) \cdot(\bar{v}-v) d u$.

Then, the key remark is that when $v, v_{*}, \theta$ are fixed, the unit vector $\sigma$ describes a sub-sphere of dimension $(N-2)$ included in $\mathbb{S}^{N-1}$, and the integral of the first order 
term over this sub-sphere is zero by gathering antipodal points. Therefore, we get

$$
I_{1,2,1} \leq C \int_{\mathbb{R}^{2 N} \times \mathbb{S}^{N-1} \times[0,1]}\left|\nabla^{2}\left(S\langle\cdot\rangle^{q}\right)\right|\left(\bar{v}_{u}\right)\left|v-v_{*}\right|^{\gamma+2}(\tan \theta / 2)^{2} b_{r}^{\varepsilon}\left|D_{*}\right| d v d v_{*} d \sigma d u \text {. }
$$

Using the same backward change of variable as in the case $\nu<1$, we deduce

$$
\begin{array}{r}
I_{1,2,1} \leq C \int_{[0,1]} \int_{\mathbb{R}^{2 N} \times \mathbb{S}^{N-1}}\left|\nabla^{2}\left(S\langle\cdot\rangle^{q}\right)(v)\right|\left|v-v_{*}\right|^{\gamma+2}(\tan \theta / 2)^{2} b_{r}^{\varepsilon}\left|D_{*}\right| d v d v_{*} d \sigma d u \\
\leq C\left(\int_{\mathbb{S}^{N-1}}(\tan \theta / 2)^{2} b_{r}^{\varepsilon} d \sigma\right)\|S\|_{W_{q+\gamma+2}^{2,1}}\|D\|_{L_{\gamma+2}^{1}},
\end{array}
$$

for some constant $C>0$ independent of $\varepsilon$. Thus, since

$$
\int_{\mathbb{S}^{N-1}}(\sin \theta / 2)^{2} b_{r}^{\varepsilon} d \sigma<+\infty
$$

we get again (for $q \geq \gamma+2$ )

$$
I_{1,2,1} \leq C C_{s}\|D\|_{L_{q}^{1}}
$$

Combining all the previous estimates, we deduce (for $q \geq 2$ when $\nu<1$ and $q \geq 4$ when $1 \leq \nu<2$ )

$$
\frac{d}{d t}\|D\|_{L_{q}^{1}} \leq C_{+}\|D\|_{L_{q}^{1}}-K_{-}\|D\|_{L_{q+\gamma}^{1}}
$$

which concludes the proof of Theorem 1.1 and Proposition 1.2 in the case when assumptions H3-1, H3-2 or H3-3 hold.

2.2. Non mollified soft potentials. We now assume that H3-4 holds (that is, in particular, $\gamma \leq 0$ ). We do not need to perform the splitting between cutoff and non-cutoff parts since large velocities are well-behaved, but another difficulty occurs because of the singularity of the kinetic collision kernel $\Phi$ for small relative velocities. We write the proof shortly, pointing out the differences with the previous subsection.

We have again

$$
\begin{aligned}
2 \int_{\mathbb{R}^{N}} Q(S, D) & \operatorname{sgn}(D)\langle v\rangle^{q} d v \\
= & \int_{\mathbb{R}^{N} \times \mathbb{R}^{N} \times \mathbb{S}^{N-1}} \Phi b\left[S_{*}^{\prime} D^{\prime}-S_{*} D\right] \operatorname{sgn}(D)\langle v\rangle^{q} d v d v_{*} d \sigma \\
& +\int_{\mathbb{R}^{N} \times \mathbb{R}^{N} \times \mathbb{S}^{N-1}} \Phi b\left[D_{*}^{\prime} S^{\prime}-D_{*} S\right] \operatorname{sgn}(D)\langle v\rangle^{q} d v d v_{*} d \sigma=: I_{1}+I_{2} .
\end{aligned}
$$

For the $I_{1}$ term again, we use Lemma 2.1 to deduce (assuming $q \geq 2$ )

$$
\left|\int_{\sigma}\left(\left\langle v^{\prime}\right\rangle^{q}-\langle v\rangle^{q}\right) b d \sigma\right| \leq \operatorname{cst}\left(\langle v\rangle^{q}+\left\langle v_{*}\right\rangle^{q}\right) .
$$


Then we compute (for $p>N /(N+\gamma)$ )

$$
\begin{aligned}
& I_{1}=\int_{v, v_{*}, \sigma}\left[S_{*}^{\prime} D^{\prime}-S_{*} D\right] \operatorname{sgn}(D)\langle v\rangle^{q}\left|v-v_{*}\right|^{\gamma} b d v d v_{*} d \sigma \\
& =\int_{v, v_{*}, \sigma} S_{*}\left[D \operatorname{sgn}\left(D^{\prime}\right)\left\langle v^{\prime}\right\rangle^{q}-|D|\langle v\rangle^{q}\right]\left|v-v_{*}\right|^{\gamma} b d v d v_{*} d \sigma \\
& \leq \int_{v, v_{*}, \sigma} S_{*}|D|\left[\left\langle v^{\prime}\right\rangle^{q}-\langle v\rangle^{q}\right]\left|v-v_{*}\right|^{\gamma} b d v d v_{*} d \sigma \\
& \leq C \int_{v, v_{*}} S_{*}|D|\left|v-v_{*}\right|^{\gamma}\left[\langle v\rangle^{q}+\left\langle v_{*}\right\rangle^{q}\right] d v d v_{*} \\
& \leq C \int D\langle v\rangle^{q}\left(\int_{\left|v-v_{*}\right| \geq 1} S_{*}+\int_{\left|v-v_{*}\right| \leq 1} S_{*}\left|v-v_{*}\right|^{\gamma}\right) \\
& +C \int D\left(\int_{\left|v-v_{*}\right| \geq 1} S_{*}\left\langle v_{*}\right\rangle^{q}+\int_{\left|v-v_{*}\right| \leq 1} S_{*}\left|v-v_{*}\right|^{\gamma}\left\langle v_{*}\right\rangle^{q}\right) \\
& \leq C \int D\langle v\rangle^{q}\left(\|S\|_{L^{1}}+\|S\|_{L^{p}}\right)+C \int D\left(\|S\|_{L_{q}^{1}}+\operatorname{cst}\langle v\rangle^{q}\|S\|_{L^{p}}\right) \\
& \leq C\left(\|S\|_{L_{q}^{1}}+\|S\|_{L^{p}}\right)\|D\|_{L_{q}^{1}} \text {. }
\end{aligned}
$$

The $I_{2}$ term writes again

$$
\begin{aligned}
I_{2}=\int_{v, v_{*}, \sigma} & {\left[D_{*}^{\prime} S^{\prime}-D_{*} S\right] \operatorname{sgn}(D)\langle v\rangle^{q}\left|v-v_{*}\right|^{\gamma} b d v d v_{*} d \sigma } \\
= & \int_{v, v_{*}, \sigma} D_{*} S\left[\operatorname{sgn}\left(D^{\prime}\right)\left\langle v^{\prime}\right\rangle^{q}-\operatorname{sgn}(D)\langle v\rangle^{q}\right]\left|v-v_{*}\right|^{\gamma} b d v d v_{*} d \sigma \\
\leq & \int_{v, v_{*}, \sigma} D_{*} \operatorname{sgn}(D)\langle v\rangle^{q}\left[\frac{S\left(\phi_{\sigma}\left(v, v_{*}\right)\right)}{\left.(\cos \theta / 2)^{N+\gamma}-S\right]\left|v-v_{*}\right|^{\gamma} b d v d v_{*} d \sigma}\right. \\
& \leq \int_{v, v_{*}}\left|D_{*}\right|\langle v\rangle^{q}\left|\int_{\mathbb{S}^{N-1}}\left(\frac{S\left(\phi_{\sigma}\left(v, v_{*}\right)\right)}{(\cos \theta / 2)^{N+\gamma}}-S\right) b d \sigma\right|\left|v-v_{*}\right|^{\gamma} d v d v_{*},
\end{aligned}
$$


where the spherical integral splits into

$$
\begin{aligned}
& \langle v\rangle^{q} \int_{\mathbb{S}^{N-1}}\left(\frac{S(\bar{v})}{(\cos \theta / 2)^{N+\gamma}}-S\right) b d \sigma \\
& =\int_{\mathbb{S}^{N-1}} \frac{S(\bar{v})\langle\bar{v}\rangle^{q}-S(v)\langle v\rangle^{q}}{(\cos \theta / 2)^{N+\gamma}} b d \sigma \\
& +\int_{\mathbb{S}^{N-1}}\left(\frac{1}{(\cos \theta / 2)^{N+\gamma}}-1\right) b d \sigma S(v)\langle v\rangle^{q}+\int_{\mathbb{S}^{N-1}}\left(\frac{\langle v\rangle^{q}-\langle\bar{v}\rangle^{q}}{(\cos \theta / 2)^{N+\gamma}}\right) S(\bar{v}) b d \sigma,
\end{aligned}
$$

which in turn yields a corresponding splitting of $I_{2}$ into three parts $I_{2,1}+I_{2,2}+I_{2,3}$.

For the $I_{2,3}$ term, we use again the change of variable of cancellation lemmas backward and we use (2.1) (for $p>N /(N+\gamma)$ ):

$$
\begin{aligned}
I_{2,3} \leq C \int_{v, v_{*}}\left|D_{*}\right| S(v)\left|v-v_{*}\right|^{\gamma}\left(\int_{\mathbb{S}^{N-1}}\left|\left\langle v^{\prime}\right\rangle^{q}-\langle v\rangle^{q}\right| b d \sigma\right) d v d v_{*} \\
\leq C\left(\|S\|_{L_{q}^{1}}+\|S\|_{L^{p}}\right)\|D\|_{L_{q}^{1}} .
\end{aligned}
$$

For the $I_{2,2}$ term, using again

$$
\left|\frac{1}{(\cos \theta / 2)^{N+\gamma}}-1\right| \leq C(1-\cos \theta),
$$

we get

$$
I_{2,2} \leq C\left(\|S\|_{L_{q}^{1}}+\|S\|_{L^{p}}\right)\|D\|_{L^{1}}
$$

Finally, for the $I_{2,1}$ term, we assume first for simplicity $\nu<1$ and we denote $\bar{v}_{u}=(1-u) v+u \bar{v}$. We Taylor-expand $S\langle\cdot\rangle^{q}$ :

$$
I_{2,1} \leq C \int_{\mathbb{R}^{2 N} \times \mathbb{S}^{N-1} \times[0,1]}\left|\nabla\left(S\langle\cdot\rangle^{q}\right)\right|\left(\bar{v}_{u}\right)\left|v-v_{*}\right|^{\gamma+1}(\tan \theta / 2) b\left|D_{*}\right| d v d v_{*} d \sigma d u .
$$

Hence we deduce

$$
I_{2,1} \leq C\left(\int_{\mathbb{S}^{N-1}}(\tan \theta / 2) b d \sigma\right)\|S\|_{W_{q+1+\gamma}^{1,1}}\|D\|_{L_{q}^{1}}
$$

when $\gamma+1 \geq 0$, and

$$
I_{2,1} \leq C\left(\int_{\mathbb{S}^{N-1}}(\tan \theta / 2) b d \sigma\right)\left(\|S\|_{W_{q}^{1,1}}+\|\nabla S\|_{L^{p}}\right)\|D\|_{L_{q}^{1}}
$$

with $p>N /(N+\gamma+1)$ else. The case $1 \leq \nu<2$ can be treated in a similar way by Taylor-expanding at second order as in the previous proof.

Combining all the previous estimates, we obtain

$$
\frac{d}{d t}\|D\|_{L_{q}^{1}} \leq C_{s}\|D\|_{L_{q}^{1}}
$$


which concludes the proof of Theorem 1.1 and Proposition 1.2.

\section{Proof of the estimates on the propagation of smoothness}

We now turn to the

Proof of Theorem 1.3: We begin the proof (Subsection 3.1) under the assumptions H1, H2 with $\nu<1$ and $(\mathbf{H 3 - 1}, \mathbf{H 3 - 2}$ or $\mathbf{H 3 - 3})$. We detail only the cases of assumptions H3-3 or H3-4, since the two other cases are similar (and somewhat simpler).

3.1. Hard potentials and mollified soft potentials. We assume that (for some $q \geq 2$ ), we have an initial datum $f(0, \cdot) \in W_{q}^{1,1}\left(\mathbb{R}^{N}\right)$, and we consider a solution $f$ to the spatially homogeneous Boltzmann equation (1.1).

We split $Q$ (and correspondingly $B, b$ ) like in the previous section into two operators $Q_{c}$ and $Q_{r}$. Then, we compute (for $q \geq 2$ ), denoting by $\partial_{v} f$ any (first order) partial derivative of $f$ with respect to one of the components of $v$, the following quantity:

$$
\begin{aligned}
\frac{d}{d t} \int_{\mathbb{R}^{N}}\left|\partial_{v} f\right|\langle v\rangle^{q} d v=\int_{\mathbb{R}^{N}} Q_{r}\left(f, \partial_{v} f\right) & \operatorname{sgn}\left(\partial_{v} f\right)\langle v\rangle^{q} d v \\
& +\int_{\mathbb{R}^{N}} Q_{c}\left(f, \partial_{v} f\right) \operatorname{sgn}\left(\partial_{v} f\right)\langle v\rangle^{q} d v:=I_{1}+I_{2} .
\end{aligned}
$$

We first consider the term $I_{2}$ corresponding to the cutoff part:

$$
\begin{gathered}
I_{2}=\int_{v, v_{*}, \sigma}\left[f_{*}^{\prime} \partial_{v} f^{\prime}+f^{\prime} \partial_{v} f_{*}^{\prime}-f_{*} \partial_{v} f-f \partial_{v} f_{*}\right] \operatorname{sgn}\left(\partial_{v} f\right)\langle v\rangle^{q} B_{c} \\
\leq \int_{v, v_{*}, \sigma}\left[f_{*}^{\prime}\left|\partial_{v} f^{\prime}\right|+f^{\prime}\left|\partial_{v} f^{\prime}\right|_{*}-f_{*}\left|\partial_{v} f\right|-f\left|\partial_{v} f_{*}\right|\right]\langle v\rangle^{q} B_{c}+2 \int_{v, v_{*}, \sigma} f\left|\partial_{v} f_{*}\right|\langle v\rangle^{q} B_{c} \\
=\int_{\mathbb{R}^{N}} Q_{c}\left(f,\left|\partial_{v} f\right|\right)\langle v\rangle^{q} d v+2 \int_{v, v_{*}, \sigma} f\left|\partial_{v} f_{*}\right|\langle v\rangle^{q} B_{c} .
\end{gathered}
$$

Arguing as in the proof of the stability estimates, we get

$$
\frac{d}{d t} \int_{\mathbb{R}^{N}}\left|\partial_{v} f\right|\langle v\rangle^{q} d v \leq C_{\varepsilon}\left\|\partial_{v} f\right\|_{L_{q}^{1}}-K\left\|\partial_{v} f\right\|_{L_{q+\gamma_{+}}^{1}}
$$

where $C_{\varepsilon}$ depends on $\|f\|_{L_{q+\gamma}^{1}}$ and $\varepsilon$ (indeed, as explained in the proof of the stability estimates, since $\nu<1$ the constant $C_{\varepsilon}$ can be taken independent of $\varepsilon$ ), and $K$ only depends on a constant $C>0$ such that

$$
\int_{\mathbb{R}^{N}} f\left|v-v_{*}\right|^{\gamma} d v_{*} \geq C\langle v\rangle^{\gamma}
$$


We now turn to the non cutoff part. We write

$$
\begin{gathered}
I_{1}=\int_{v, v_{*}, \sigma}\left[f_{*}^{\prime} \partial_{v} f^{\prime}+f^{\prime} \partial_{v} f_{*}^{\prime}-f_{*} \partial_{v} f-f \partial_{v} f_{*}\right] \operatorname{sgn}\left(\partial_{v} f\right)\langle v\rangle^{q} B_{r} \\
=\int_{v, v_{*}, \sigma}\left[\operatorname{sgn}\left(\partial_{v} f\right)^{\prime}\left\langle v^{\prime}\right\rangle^{q}+\operatorname{sgn}\left(\partial_{v} f\right)_{*}^{\prime}\left\langle v_{*}^{\prime}\right\rangle^{q}-\operatorname{sgn}\left(\partial_{v} f\right)\langle v\rangle^{q}-\operatorname{sgn}\left(\partial_{v} f\right)_{*}\left\langle v_{*}\right\rangle^{q}\right] f_{*} \partial_{v} f B_{r} \\
\leq \int_{v, v_{*}, \sigma}\left[\operatorname{sgn}\left(\partial_{v} f\right)\left(v_{*}^{\prime}\right)-\operatorname{sgn}\left(\partial_{v} f\right)\left(v_{*}\right)\right] f_{*} \partial_{v} f(v)\left\langle v_{*}\right\rangle^{q} B_{r} \\
+\int_{v, v_{*}}\left|\int_{\sigma}\left[\left\langle v^{\prime}\right\rangle^{q}-\langle v\rangle^{q}\right] b_{r} d \sigma\right|\left(f_{*}\left|\partial_{v} f(v)\right|+f\left|\partial_{v} f\left(v_{*}\right)\right|\right)\left|v-v_{*}\right|^{\gamma}=: I_{1,1}+I_{1,2} .
\end{gathered}
$$

The term $I_{1,2}$ is easily controlled thanks to Lemma 2.1.

$$
I_{1,2} \leq \delta(\varepsilon)\left\|\partial_{v} f\right\|_{L_{q+\gamma}^{1}}
$$

with $\delta(\varepsilon) \rightarrow 0$ as $\varepsilon \rightarrow 0$.

We now focus on the most difficult term $I_{1,1}$. Since the proof makes use, in the sequel, of an intricate kind of integration by parts, we write down first the simple case when the dimension is $N=2$, for the sake of clarity.

In this case $(N=2)$, we define the change of variables $v_{*} \mapsto w$ (for given $v, \theta$ ), where

$$
w=v_{*}^{\prime}=\frac{v+v_{*}}{2}-R_{\theta}\left(\frac{v-v_{*}}{2}\right)
$$

$\left(R_{\theta}\right.$ denotes the rotation of angle $\theta$ ), whose Jacobian determinant is clearly $(\cos \theta / 2)^{2}$ and which can be inverted in

$$
v_{*}=v_{*}(v, w, \theta)=\frac{R_{-\frac{\theta}{2}} w+\sin \frac{\theta}{2} R_{\frac{\pi}{2}} v}{\cos \frac{\theta}{2}} .
$$

Using this change of variables in the first part of the integral (and the fact that $\left.|v-w|=\cos \theta / 2\left|v-v_{*}\right|\right)$, we obtain

$$
\begin{gathered}
I_{1,1} \leq \int_{v \in \mathbb{R}^{2}} \int_{w \in \mathbb{R}^{2}} \int_{\theta=0}^{\varepsilon} \operatorname{sgn}\left(\partial_{v} f\right)(w) \partial_{v} f(v) b_{r}(\cos \theta) \\
\times\left\{f\left(v_{*}(v, w, \theta)\right)\left\langle v_{*}(v, w, \theta)\right\rangle^{q}\left|v-v_{*}(v, w, \theta)\right|^{\gamma}\left(\cos \frac{\theta}{2}\right)^{-2}\right. \\
\left.-f(w)\langle w\rangle^{q}|v-w|^{\gamma}\right\} d \theta d w d v \\
\leq \int_{v \in \mathbb{R}^{2}} \int_{w \in \mathbb{R}^{2}} \int_{\theta=0}^{\varepsilon} \operatorname{sgn}\left(\partial_{v} f\right)(w) \partial_{v} f(v) b_{r}(\cos \theta)
\end{gathered}
$$




$$
\begin{gathered}
\times\left[\left(\cos \frac{\theta}{2}\right)^{-2-\gamma}-1\right] f(w)\langle w\rangle^{q}|v-w|^{\gamma} d \theta d w d v \\
+\int_{v \in \mathbb{R}^{2}} \int_{w \in \mathbb{R}^{2}} \int_{\theta=0}^{\varepsilon} \operatorname{sgn}\left(\partial_{v} f\right)(w) \partial_{v} f(v) b_{r}(\cos \theta)\left(\cos \frac{\theta}{2}\right)^{-2-\gamma} \\
\times\left\{f\left(v_{*}(v, w, \theta)\right)-f(w)\right\}\langle w\rangle^{q}|v-w|^{\gamma} d \theta d w d v \\
+\int_{v \in \mathbb{R}^{2}} \int_{w \in \mathbb{R}^{2}} \int_{\theta=0}^{\varepsilon} \operatorname{sgn}\left(\partial_{v} f\right)(w) \partial_{v} f(v) b_{r}(\cos \theta)\left(\cos \frac{\theta}{2}\right)^{-2-\gamma} \\
\times\left\{\left\langle v_{*}(v, w, \theta)\right\rangle^{q}-\langle w\rangle^{q}\right\} f\left(v_{*}(v, w, \theta)\right)|v-w|^{\gamma} d \theta d w d v=: I_{1,1,1}+I_{1,1,2}+I_{1,1,3} .
\end{gathered}
$$

The first term $I_{1,1,1}$ is controlled thanks to

$$
\left|\left(\cos \frac{\theta}{2}\right)^{-2-\gamma}-1\right| \leq C(1-\cos \theta)
$$

which yields

$$
I_{1,1,1} \leq \operatorname{cst}\left\|\partial_{v} f\right\|_{L_{q}^{1}}
$$

The third term $I_{1,1,3}$ is controlled thanks to the argument of Lemma 2.1]

$$
I_{1,1,3} \leq \delta(\varepsilon)\left\|\partial_{v} f\right\|_{L_{q+\gamma}^{1}} .
$$

Finally we use integration by parts (according to $v$ ) on the second term $I_{1,1,2}$ (and the fact that the differential in $v$ of $v_{*}(v, w, \theta)$ has bound $\left.\tan \theta / 2\right)$ :

$$
\begin{aligned}
& I_{1,1,2} \leq \int_{v \in \mathbb{R}^{2}} \int_{w \in \mathbb{R}^{2}} \int_{\theta=0}^{\varepsilon} b_{r}(\cos \theta)\left(\cos \frac{\theta}{2}\right)^{-3-\gamma}\left(\sin \frac{\theta}{2}\right) f(v) \\
& \times\left|\partial_{v} f\right|\left(v_{*}(v, w, \theta)\right)|v-w|^{\gamma}\langle w\rangle^{q} d \theta d w d v \\
&+ \gamma \int_{v \in \mathbb{R}^{2}} \int_{w \in \mathbb{R}^{2}} \int_{\theta=0}^{\varepsilon} b_{r}(\cos \theta)\left(\cos \frac{\theta}{2}\right)^{-2-\gamma} f(v) \\
& \times\left|f\left(v_{*}(v, w, \theta)\right)-f(w)\right||v-w|^{\gamma-1}\langle w\rangle^{q} d \theta d w d v \\
& \leq \int_{v \in \mathbb{R}^{2}} \int_{v_{*} \in \mathbb{R}^{2}} \int_{\theta=0}^{\varepsilon} b_{r}(\cos \theta)\left(\tan \frac{\theta}{2}\right) f(v)\left\langle v_{*}^{\prime}\right\rangle^{q}\left|v-v_{*}\right|^{\gamma}\left|\partial_{v} f\left(v_{*}\right)\right| d \theta d v_{*} d v \\
& \int_{v \in \mathbb{R}^{2}} \int_{v_{*} \in \mathbb{R}^{2}} \int_{\theta=0}^{\varepsilon} \int_{u=0}^{1} b_{r}(\cos \theta)\left(\cos \frac{\theta}{2}\right)^{-3-\gamma}\left(\sin \frac{\theta}{2}\right) f(v)|v-w|^{\gamma}\langle w\rangle^{q} \\
& \times\left|\partial_{v} f\right|\left((1-u) w+u v_{*}(v, w, \theta)\right) d u d \theta d w d v \\
& \leq \delta(\varepsilon)\left\|\partial_{v} f\right\|_{L_{q+\gamma}^{1}},
\end{aligned}
$$

where $\lim _{\varepsilon \rightarrow 0} \delta(\varepsilon)=0$. Here, we have assumed that $\|f\|_{L_{q+\gamma}^{1}}$ is bounded (this norm is known to be at least propagated, Cf. [7] for example). 
Then, using estimate (3.1), we see that (optimizing $\varepsilon$ ), the proof of the theorem is complete.

Let us now explain how to deal with the general case of dimension $N \geq 2$.

In the formula for $I_{1,1}$

$$
I_{1,1}=\int_{v, v_{*}, \sigma}\left[\operatorname{sgn}\left(\partial_{v} f\right)\left(v_{*}^{\prime}\right)-\operatorname{sgn}\left(\partial_{v} f\right)\left(v_{*}\right)\right] f_{*} \partial_{v} f(v)\left\langle v_{*}\right\rangle^{q} B_{r},
$$

we use the change of variable $v_{*} \mapsto w$ (for given $v, \sigma$ ) where

$$
w=v_{*}^{\prime}=\frac{v+v_{*}}{2}-\frac{\left|v-v_{*}\right|}{2} \sigma,
$$

which can be inverted in

$$
v_{*}=v_{*}(v, w, \sigma)=2 w-v+\frac{|v-w|^{2}}{(v-w) \cdot \sigma} \sigma
$$

and whose Jacobian determinant is

$$
J=2^{N-1} \frac{|v-w|^{2}}{((v-w) \cdot \sigma)^{2}} .
$$

We get

$$
\begin{aligned}
I_{1,1} & \leq\left|\int_{v, w, \sigma} \operatorname{sgn}\left(\partial_{v} f\right)(w) f\left(v_{*}(v, w, \sigma)\right) \partial_{v} f(v)\left(\frac{|v-w|^{2}}{(v-w) \cdot \sigma}\right)^{\gamma}\right| v-\left.w\right|^{\gamma} \\
& \times\left\langle v_{*}(v, w, \sigma)\right\rangle^{q} b_{r}\left(-1+2\left(\frac{v-w}{|v-w|} \cdot \sigma\right)^{2}\right) \frac{2^{N-1}}{\left(\frac{v-w}{|v-w|} \cdot \sigma\right)^{2}} d \sigma d w d v \\
& -\int_{v, w, \sigma} \operatorname{sgn}\left(\partial_{v} f\right)(w) f(w) \partial_{v} f(v)|v-w|^{\gamma}\langle w\rangle^{q} b_{r}\left(\frac{v-w}{|v-w|} \cdot \sigma\right) d \sigma d w d v \mid,
\end{aligned}
$$

and using the decomposition of the unit vector $\sigma$ :

$$
\sigma=\cos \theta \frac{v-w}{|v-w|}+\sin \theta n
$$

where $n \in S^{N-1} \cap(v-w)^{\perp}$ and $0 \leq \theta \leq \varepsilon$ (we look here to the non cutoff part of the cross-section), we end up with

$$
\begin{aligned}
& I_{1,1} \leq \mid \int_{v, w} \int_{\theta=0}^{\varepsilon} \int_{n \in S^{N-1} \cap(v-w)^{\perp}} \operatorname{sgn}\left(\partial_{v} f\right)(w) f(w+\tan \theta|v-w| n) \partial_{v} f(v) \\
& \times|v-w|^{\gamma}\langle w+\tan \theta|v-w| n\rangle^{q} \frac{b_{r}(\cos (2 \theta))}{\cos ^{2+\gamma} \theta} 2^{N-1} \sin ^{N-2} \theta d n d \theta d w d v \\
& \quad-\int_{v, w} \int_{\theta=0}^{\varepsilon} \int_{n \in S^{N-1} \cap(v-w)^{\perp}} \operatorname{sgn}\left(\partial_{v} f\right)(w) f(w) \partial_{v} f(v)|v-w|^{\gamma}
\end{aligned}
$$




$$
\times\langle w\rangle^{q} b_{r}(\cos \theta) \sin ^{N-2} \theta d n d \theta d w d v \mid .
$$

After integration by part (in $v$ ), and the use of the change of variables $\delta=2 \theta$ in the first term, we obtain

$$
\begin{gathered}
I_{1,1} \leq\left|\int_{v, w} \int_{\delta=0}^{\varepsilon} \int_{n \in S^{N-1} \cap(v-w)^{\perp}} \operatorname{sgn}\left(\partial_{v} f\right)(w)\right| v-\left.w\right|^{\gamma} \\
{\left[f(w+\tan \delta / 2|v-w| n)\left(\cos \frac{\delta}{2}\right)^{-\gamma-N}\langle w+\tan \delta / 2|v-w| n\rangle^{q}-f(w)\langle w\rangle^{q}\right]} \\
+\left|\int_{v, w} \int_{\delta=\varepsilon}^{2 \varepsilon} \int_{n \in S^{N-1} \cap(v-w)^{\perp}} \operatorname{sgn}\left(\partial_{v} f\right)(w)\right| v-\left.w\right|^{\gamma}\langle w+\tan \delta / 2|v-w| n\rangle^{q} \\
\times f(w+\tan \delta / 2|v-w| n)\left(\partial_{v} f\right)(v)\left(\cos \frac{\delta}{2}\right)^{-\gamma-N} \sin ^{N-2} \delta d n d \delta d w d v \mid \\
=: I_{1,1,1}+I_{1,1,2}+I_{1,1,3} .
\end{gathered}
$$

Then for the first term $I_{1,1,1}$ we have

$$
\begin{gathered}
I_{1,1,1} \leq \gamma\left|\int_{v, w} \int_{\delta=0}^{\varepsilon} \int_{n \in S^{N-1} \cap(v-w)^{\perp}} f(v) \operatorname{sgn}\left(\partial_{v} f\right)(w)\right| v-\left.w\right|^{\gamma-1} \\
{\left[f(w+\tan \delta / 2|v-w| n)\left(\cos \frac{\delta}{2}\right)^{-\gamma-N}\langle w+\tan \delta / 2|v-w| n\rangle^{q}-f(w)\langle w\rangle^{q}\right]} \\
b_{r}(\cos \delta) \sin ^{N-2} \delta d n d \delta d w d v \mid
\end{gathered}
$$

from which we deduce straightforwardly as before

$$
I_{1,1,1} \leq \delta(\varepsilon)\left\|\partial_{v} f\right\|_{L_{q+\gamma}^{1}}
$$

by using the control on $\left(\cos ^{-\gamma-N} \delta / 2-1\right)$ and Taylor-expanding $f$ and $\langle\cdot\rangle^{q}$.

For the second term $I_{1,1,2}$ we have

$$
\begin{aligned}
& I_{1,1,2} \leq \mid\left|\int_{v, w} \int_{\delta=0}^{\varepsilon} f(v) \operatorname{sgn}\left(\partial_{v} f\right)(w)\right| v-\left.w\right|^{\gamma} \\
& \times \partial_{v}\left(\int_{n \in S^{N-1} \cap(v-w)^{\perp}}\left(f\langle\cdot\rangle^{q}\right)(w+\tan \delta / 2|v-w| n) d n\right) \\
& \quad \times\left(\cos \frac{\delta}{2}\right)^{-\gamma-N} b_{r}(\cos \delta) \sin ^{N-2} \delta d \delta d w d v \mid
\end{aligned}
$$




$$
\begin{aligned}
& \leq \operatorname{cst}\left|\int_{v, w} \int_{\delta=0}^{\varepsilon} f(v) \operatorname{sgn}\left(\partial_{v} f\right)(w)\right| v-\left.w\right|^{\gamma} \\
& \times\left(\int_{n \in S^{N-1} \cap(v-w)^{\perp}}\left|\partial_{v}\left(f\langle\cdot\rangle^{q}\right)\right|(w+\tan \delta / 2|v-w| n) d n\right) \\
& \times\left(\cos \frac{\delta}{2}\right)^{-\gamma-N} \tan \delta / 2 b_{r}(\cos \delta) \sin ^{N-2} \delta d \delta d w d v \mid
\end{aligned}
$$

(the derivative taken on a sphere depending on $v$ has been treated by taking local coordinates) from which we deduce

$$
I_{1,1,2} \leq \delta(\varepsilon)\left\|\partial_{v} f\right\|_{L_{q+\gamma}^{1}}
$$

For the third term we have

$$
\begin{gathered}
I_{1,1,3} \leq \mid \int_{v, w} \int_{\delta=\varepsilon}^{2 \varepsilon} \int_{n \in S^{N-1} \cap(v-w)^{\perp}} \\
\partial_{v} f(v) \operatorname{sgn}\left(\partial_{v} f\right)(w)|v-w|^{\gamma}\langle w+\tan \delta / 2|v-w| n\rangle^{q} f(w+\tan \delta / 2|v-w| n) \\
\times\left(\cos \frac{\delta}{2}\right)^{-\gamma-2} b_{r}(\cos \delta) \sin ^{N-2} \delta d n d \delta d w d v \mid
\end{gathered}
$$

from which we deduce thanks to the cutoff (by coming back to the classical variables)

$$
I_{1,1,3} \leq C_{\varepsilon}\left\|\partial_{v} f\right\|_{L_{\gamma}^{1}}
$$

(for some constant $C_{\varepsilon}$ blowing-up as $\varepsilon \rightarrow 0$ ).

These estimates (where we have used the boundedness of weighted $L^{1}$ norms like in dimension 2) enables to complete the proof of Theorem 1.3 (for hard and smoothed soft potentials) also in dimension bigger than 2 .

3.2. Non mollified soft potentials. We now prove Theorem 1.3 under assumptions $\mathbf{H 1}, \mathbf{H} 2$ with $\nu<1$ and $\mathbf{H 3 - 4}$. The additional difficulty here is the singularity of the collision kernel for small relative velocity. As pointed out in the proofs of stability estimates, this suggests to use some $L^{p}$ norm with $p>N /(N+\gamma)$ of $f$, in order to control some convolution terms of the form

$$
v \mapsto \int_{\left|v-v_{*}\right| \leq 1}\left|v-v_{*}\right|^{\gamma} f\left(v_{*}\right) d v_{*} .
$$

We first state a result showing that we are able to propagate $L^{1}$ norms of the gradient as soon as we have some time integrability of some $L^{p}$ and $L^{1}$ moments. 
Proposition 3.1. Let $B$ be a collision kernel satisfying assumptions $\mathbf{H 1}$, H2 with $\nu<1$ and H3-4. Let $f$ be a solution on $[0, T]$ to the corresponding spatially homogeneous Boltzmann equation (1.1). Suppose that for $p>N /(N+\gamma)$ and $q \geq 2$, one has $f \in L^{1}\left([0, T] ; L^{p} \cap L_{q}^{1}\left(\mathbb{R}^{N}\right)\right)$. Assume also that $f(0, \cdot) \in W_{q}^{1,1}\left(\mathbb{R}^{N}\right)$. Then $f \in L^{\infty}\left([0, T] ; W_{q}^{1,1}\left(\mathbb{R}^{N}\right)\right)$.

Proof of proposition 3.1. We compute (for $q \geq 2$ )

$$
\begin{gathered}
\frac{d}{d t} \int\left|\partial_{v} f\right|\langle v\rangle^{q} d v=\int_{v, v_{*}, \sigma}\left[f_{*}^{\prime} \partial_{v} f^{\prime}+f^{\prime} \partial_{v} f_{*}^{\prime}-f_{*} \partial_{v} f-f \partial_{v} f_{*}\right] \operatorname{sgn}\left(\partial_{v} f\right)\langle v\rangle^{q} B \\
\leq \int_{v, v_{*}, \sigma}\left[\operatorname{sgn}\left(\partial_{v} f\right)\left(v_{*}^{\prime}\right)-\operatorname{sgn}\left(\partial_{v} f\right)\left(v_{*}\right)\right] f_{*} \partial_{v} f\left\langle v_{*}\right\rangle^{q} B \\
+\int_{v, v_{*}}\left(f_{*}\left|\partial_{v} f(v)\right|+f\left|\partial_{v} f\left(v_{*}\right)\right|\right)\left|\int_{\sigma}\left[\left\langle v^{\prime}\right\rangle^{q}-\langle v\rangle^{q}\right]\right| B \quad=: I_{1}+I_{2} .
\end{gathered}
$$

The term $I_{2}$ is immediately controlled by Lemma 2.1 .

$$
I_{2} \leq C\left\|\partial_{v} f\right\|_{L_{q}^{1}}\left(\|f\|_{L^{1}}+\|f\|_{L^{p}}\right)
$$

for some constant $C>0$. Then we focus on the term $I_{1}$ and we split it as $I_{1}^{c}+I_{1}^{r}$ according to the decomposition of the collision kernel $B=B_{c}+B_{r}$ as before.

We first note that (for some constant $C_{\varepsilon}$ possibly blowing-up when $\varepsilon \rightarrow 0$ )

$$
\begin{gathered}
I_{1}^{c} \leq\left|\int_{v, v_{*}, \sigma}\left[\operatorname{sgn}\left(\partial_{v} f\right)\left(v_{*}^{\prime}\right)-\operatorname{sgn}\left(\partial_{v} f\right)\left(v_{*}\right)\right] f_{*} \partial_{v} f\left\langle v_{*}\right\rangle^{q} B_{c}\right| \\
\leq C_{\varepsilon}\left\|\partial_{v} f\right\|_{L_{q}^{1}}\left(\|f\|_{L^{1}}+\|f\|_{L^{p}}\right) .
\end{gathered}
$$

Then, we only detail the treatment of the non cutoff case in the simple case when the dimension is $N=2$ (the case of dimension $N>2$ can be treated like in Subsection 3.1, where hard potentials are considered). Using the change of variables 
$v_{*} \mapsto w($ for a given $v, \theta$ ), we end up with

$$
\begin{aligned}
& I_{1}^{r} \leq \mid \int_{v \in \mathbb{R}^{2}} \int_{w \in \mathbb{R}^{2}} \int_{\theta=0}^{\varepsilon} \operatorname{sgn}\left(\partial_{v} f\right)(w) \partial_{v} f(v)\langle w\rangle^{q} \\
& \times b_{r}(\cos \theta)\left(\left(\cos \frac{\theta}{2}\right)^{-2-\gamma}-1\right) f(w)|v-w|^{\gamma} d \theta d w d v \mid \\
&+\mid \int_{v \in \mathbb{R}^{2}} \int_{w \in \mathbb{R}^{2}} \int_{\theta=0}^{\varepsilon} \operatorname{sgn}\left(\partial_{v} f\right)(w) \partial_{v} f(v) b_{r}(\cos \theta)\left(\cos \frac{\theta}{2}\right)^{-2-\gamma} \\
& \times\left\{f\left(v_{*}(v, w, \theta)\right)\left\langle v_{*}(v, w, \theta)\right\rangle^{q}-f(w)\langle w\rangle^{q}\right\}|v-w|^{\gamma} d \theta d w d v \mid \\
& \leq \operatorname{cst} \int_{v \in \mathbb{R}^{2}} \int_{w \in \mathbb{R}^{2}}\left|\partial_{v} f(v)\right|\langle w\rangle^{q} f(w)|v-w|^{\gamma} d w d v \\
&+ \int_{v \in \mathbb{R}^{2}} \int_{w \in \mathbb{R}^{2}} \int_{\theta=0}^{\varepsilon} b_{r}(\cos \theta)\left(\cos \frac{\theta}{2}\right)^{-2-\gamma} \sin \frac{\theta}{2} \\
& \quad \times f(v)\left|\partial_{v}\left(f\langle\cdot\rangle^{q}\right)\right|\left(v_{*}(v, w, \theta)\right)|v-w|^{\gamma} d \theta d w d v \\
&+|\gamma| \int_{v \in \mathbb{R}^{2}} \int_{w \in \mathbb{R}^{2}} \int_{\theta=0}^{\varepsilon} b_{r}(\cos \theta)\left(\cos \frac{\theta}{2}\right)^{-2-\gamma} f(v) \\
& \quad \times\left|f\left(v_{*}(v, w, \theta)\right)\left\langle v_{*}(v, w, \theta)\right\rangle^{q}-f(w)\langle w\rangle^{q}\right||v-w|^{\gamma-1} d \theta d w d v
\end{aligned}
$$

and we deduce (using Taylor expansion as before on the last term)

$$
I_{1}^{r} \leq \operatorname{cst}\left\|\partial_{v} f\right\|_{L_{q}^{1}}\left(\|f\|_{L_{q}^{1}}+\|f\|_{L^{p}}\right)
$$

which concludes the proof of the proposition by some Gronwall argument.

Then, the $L^{p}$ norms can easily be shown to be locally propagated in time by direct energy methods:

Proposition 3.2. Let $B$ be a collision kernel satisfying assumptions $\mathbf{H 1}$, H2 with $\nu<1$ and H3-4. Let $f$ be a solution on $[0, T]$ to the corresponding spatially homogeneous Boltzmann equation (1.1). Suppose that $f(0, \cdot) \in L^{p}\left(\mathbb{R}^{N}\right)$ for some $p \in] N /(N+\gamma),+\infty]$. Then, there exists a time $\left.\left.T^{*} \in\right] 0, T\right]$ explicitly computable such that $f \in L^{\infty}\left(\left[0, T^{*}\right] ; L^{p}\left(\mathbb{R}^{N}\right)\right)$.

Proof of proposition 3.2. We compute for some $N /(N+\gamma)<p<+\infty$ (and with $C$ denoting a constant which does not depend on $p$ )

$$
\frac{d}{d t} \int_{v \in \mathbb{R}^{N}}|f(t, v)|^{p} d v=p \int_{v, v_{*}, \sigma}\left(f^{p-1} f^{\prime} f^{\prime *}-f^{p} f^{*}\right) B
$$




$$
\begin{gathered}
=p \int_{v, v_{*}, \sigma}\left(\left(f^{\prime}\right)^{p-1} f f^{*}-f^{p} f^{*}\right) B \\
\leq p \int_{v, v_{*}, \sigma}\left(\frac{1}{p} f^{p}+\left(1-\frac{1}{p}\right)\left(f^{\prime}\right)^{p}-f^{p}\right) f^{*} B \\
\leq(p-1) \int_{v, v_{*}, \sigma}\left(\left(f^{\prime}\right)^{p}-f^{p}\right) f^{*} B \\
\leq C(p-1) \int_{\theta=0}^{\pi / 2}\left(\left(\cos \frac{\theta}{2}\right)^{-2+\gamma}-1\right) b(\cos \theta) d \theta \int_{v, v_{*}} f^{p} f^{*}\left|v-v_{*}\right|^{\gamma} \\
\leq C(p-1) \int_{v} f^{p}\left(\int_{\left|v_{*}-v\right| \leq 1} f^{*}\left|v-v_{*}\right|^{\gamma} d v_{*}+\int_{\left|v_{*}-v\right| \geq 1} f^{*}\left|v-v_{*}\right|^{\gamma} d v_{*}\right) d v \\
\leq C(p-1) \int_{v} f^{p}\left[C\left(\int\left(f^{*}\right)^{p}\right)^{1 / p}+f^{*}\right] \\
\leq C(p-1)\left[\left(\int f^{p}\right)^{1+1 / p}+\int^{p}\right]
\end{gathered}
$$

(remember in theses computations that the collision kernel $B$ is taken in symmetrized form $B_{\text {sym }}$ with support in $\left.[0, \pi / 2]\right)$. As a consequence,

$$
\frac{d}{d t}\|f\|_{L^{p}} \leq C\left(1-\frac{1}{p}\right)\left(\|f\|_{L^{p}}+\|f\|_{L^{p}}^{2}\right),
$$

and we get also (passing to the limit when $p \rightarrow+\infty$ ),

$$
\frac{d}{d t}\|f\|_{L^{\infty}} \leq C\left(\|f\|_{L^{\infty}}+\|f\|_{L^{\infty}}^{2}\right) .
$$

This ends the proof of Proposition 3.2

Finally, the $L^{1}$ moments can be shown to be locally propagated, using the previous $L^{p}$ estimates in case of strong singularity at small relative velocity:

Proposition 3.3. Let $B$ be a collision kernel satisfying assumptions $\mathbf{H 1}$, H2 with $\nu<1$ and $\mathbf{H 3 - 4}$. Let $f$ be a solution on $[0, T]$ to the corresponding spatially homogeneous Boltzmann equation (1.1).

Suppose first that $\gamma+1 \geq 0$. Then for any $q \geq 2$, if $f(0, \cdot) \in L_{q}^{1}\left(\mathbb{R}^{N}\right)$, one has $f \in L^{\infty}\left([0, T] ; L_{q}^{1}\left(\mathbb{R}^{N}\right)\right)$.

Suppose then that $\gamma+2 \geq 0$. Then for any $q \geq 4$, if $f(0, \cdot) \in L_{q}^{1}\left(\mathbb{R}^{N}\right)$, one has $f \in L^{\infty}\left([0, T] ; L_{q}^{1}\left(\mathbb{R}^{N}\right)\right)$. 
Suppose finally that $\gamma+2<0$ and $f(0, \cdot) \in L^{p}\left(\mathbb{R}^{N}\right) \cap L_{q}^{1}\left(\mathbb{R}^{N}\right)$ for some $N /(N+$ $\gamma+2)<p \leq+\infty, q \geq 4$. Then, there exists a time $\left.\left.T^{*} \in\right] 0, T\right]$ explicitly computable such that $f \in L^{\infty}\left(\left[0, T^{*}\right] ; L^{p}\left(\mathbb{R}^{N}\right) \cap L_{q}^{1}\left(\mathbb{R}^{N}\right)\right)$.

Proof of proposition 3.3. We compute (for $q \geq 2$ )

$$
\begin{aligned}
& \frac{d}{d t} \int_{v \in \mathbb{R}^{N}} f(t, v)\langle v\rangle^{q} d v=\int_{v, v_{*}, \sigma}\langle v\rangle^{q}\left(f^{\prime} f^{*}-f f^{*}\right) B \\
& \quad \leq \int_{v, v_{*}}\left[\int_{\sigma}\left[\left\langle v^{\prime}\right\rangle^{q}-\langle v\rangle^{q}\right] b d \sigma\right] f f^{*}\left|v-v_{*}\right|^{\gamma} .
\end{aligned}
$$

Using Lemma 2.1, we get for $q \geq 2$ :

$$
\frac{d}{d t} \int_{v \in \mathbb{R}^{N}} f(t, v)\langle v\rangle^{q} d v \leq \operatorname{cst} \int_{v, v_{*}}\left[\left\langle v_{*}\right\rangle^{q-1}+\langle v\rangle^{q-1}\right] f f^{*}\left|v-v_{*}\right|^{\gamma+1}
$$

and for $q \geq 4$ :

$$
\frac{d}{d t} \int_{v \in \mathbb{R}^{N}} f(t, v)\langle v\rangle^{q} d v \leq \operatorname{cst} \int_{v, v_{*}}\left[\left\langle v_{*}\right\rangle^{q-2}+\langle v\rangle^{q-2}\right] f f^{*}\left|v-v_{*}\right|^{\gamma+2} .
$$

Hence this is controlled by

$$
\text { cst }\|f\|_{L_{q}^{1}}\|f\|_{L^{1}}
$$

if $q \geq 2$ and $\gamma+1 \geq 0$, or $q \geq 4$ and $\gamma+2 \geq 0$ (which concludes the proof of Proposition 3.3 in those cases immediately by some Gronwall argument). It is also controlled by

$$
\operatorname{cst}\|f\|_{L_{q}^{1}}\left(\|f\|_{L^{1}}+\|f\|_{L^{p}}\right)
$$

with $p>N /(N+\gamma+2)$ if $q \geq 4$ and $\gamma+2<0$. This concludes also the proof of Proposition 3.3 in this case: using Proposition 3.2. we see that the $L_{q}^{1}\left(\mathbb{R}^{N}\right)$ norm of $f(\cdot)$ is uniformly bounded on $\left[0, T^{*}\right]$, where $T^{*}$ is the same as in Proposition 3.2 .

In order to conclude the proof of Theorem 1.3, it remains to prove that the $L^{p}$ estimate is global in time in the case when $\gamma \in(-\nu, 0]$.

In order to do so, we shall use the regularizing effect of non cutoff interactions. The entropy a priori estimate ensures that the entropy production associated to the solution $f$ of equation (1.1) is bounded in $L^{1}([0, T]$ ) (uniformly with respect to $T \in[0,+\infty))$. This means that

$$
\int_{0}^{T} \int_{v, v_{*}, \sigma} f_{*} f \log \left(f / f^{\prime}\right) B<+\infty .
$$


Since the entropy production is monotonous (increasing) with respect to the crosssection $B$, we see that

$$
\int_{0}^{T} \int_{v, v_{*}, \sigma} f_{*} f \log \left(f / f^{\prime}\right) \max (B, 1)<+\infty .
$$

Using the results in [1], we get

$$
\int_{0}^{T}\|\sqrt{f}\|_{H^{\nu / 2}(|v| \leq R)}^{2} d t \leq C_{R}\left(1+\|f\|_{L^{1}}^{2} T\right) .
$$

By tracking the constant in the proof of this estimate, one finds $C_{R}=\operatorname{cst} R^{|\gamma|}$. Using a Sobolev embedding (remember that the Sobolev constant does not depend on $R$ ), one deduces

$$
\int_{0}^{T}\|\sqrt{f}\|_{L^{2 N /(N-\nu)}(|v| \leq R)}^{2} d t \leq \operatorname{cst} R^{|\gamma|}(1+T),
$$

and finally

$$
\int_{0}^{T}\|f\|_{L^{N /(N-\nu)}(|v| \leq R)} d t \leq \operatorname{cst} R^{|\gamma|}(1+T) .
$$

We now use a dyadic decomposition of the velocity space. For any $k \geq 0$, we have

$$
\int_{0}^{T}\|f\|_{L^{N /(N-\nu)}\left(2^{k} \leq|v| \leq 2^{k+1}\right)} d t \leq C 2^{k|\gamma|}(1+T) .
$$

Therefore, for any $\alpha \in \mathbb{R}$,

$$
\int_{0}^{T}\|f\|_{L_{\gamma-\alpha}^{N /(N-\nu)}\left(2^{k} \leq|v| \leq 2^{k+1}\right)} d t \leq C 2^{-\alpha k}(1+T) .
$$

By summing this estimate for $k=0, \ldots,+\infty$, we get when $\alpha>0$ :

$$
\int_{0}^{T}\|f\|_{L_{\gamma-\alpha}^{N /(N-\nu)}\left(\mathbb{R}^{N}\right)} d t \leq C(1+T) .
$$

We now use the fact that $\gamma>-\nu$. Since in particular $\gamma>-2$, we can use Proposition 3.3 in order to propagate the $L_{q}^{1}$ moments for any $q \geq 4$, and get

$$
\int_{0}^{T}\|f\|_{L_{q}^{1}\left(\mathbb{R}^{N}\right)} d t \leq C(1+T) .
$$

By interpolation between estimates (3.2) and (3.3), we see that

$$
\int_{0}^{T}\|f\|_{L^{p}\left(\mathbb{R}^{N}\right)} d t \leq C(1+T)
$$

for any $1<p<N /(N-\nu)$ (if $f(0, \cdot) \in L_{q}^{1}$ with $q$ big enough). But $N /(N+\gamma)<$ $N /(N-\nu)$ (since $\gamma>-\nu)$, so that we can take $p>N /(N+\gamma)$, and the assumptions 
of Proposition 3.1 hold for all times. Finally, the required smoothness is global in time.

Remark: Note that the threshold $\gamma=-\nu$ in this proof does not seem to be only a coincidence. Indeed, as explained in the introduction, for inverse power-laws interaction potentials in dimension 3, it corresponds to moderately soft potentials, that is potentials $V(r)=r^{-s}$ with $s>2$. This critical value also corresponds to the threshold below which there is no spectral gap for the linearized operator (and therefore no expected exponential relaxation rate towards equilibrium), below which it is not known how to show slowly growing bounds on the $L^{1}$ moments (that is growing more slowly than exponentially), below which it is not known how to build global smooth solutions (as pointed out in this paper). Note that Fournier in [13] also has the same threshold.

An interesting issue to be explored is to relate this focus point of so many mathematical difficulties to the physical considerations: the threshold $s=2$ is called "Manev interaction" in the physical literature (see for instance [4, 17]), and a dimensional analysis in [4] shows that for $s>2$, the Boltzmann collision term prevail on the mean-field term, whereas for $1<s<2$, the Boltzmann collision term should be negligible in front of the mean-field term.

\section{REFERENCES}

[1] Alexandre, R., Desvillettes, L., Villani, C., Wennberg, B. Entropy dissipation and long-range interactions. Arch. Ration. Mech. Anal. 152 (2000), 327-355.

[2] Arkeryd, L. On the Boltzmann equation. Arch. Rational Mech. Anal. 45 (1972), 1-34.

[3] Bobylev, A. V., Cercignani, C. On the rate of entropy production for the Boltzmann equation. J. Statist. Phys. 94, 3-4 (1999), 603-618.

[4] Bobylev, A. V., Dukes, P., Illner, R., Victory, H. D. On Vlasov-Manev equations. I. Foundations, properties, and nonglobal existence. J. Statist. Phys. 88 3-4 (1997), 885-911.

[5] Cercignani, C. The Boltzmann equation and its applications. Springer-Verlag, New York, 1988.

[6] Cercignani, C., Illner, R., Pulvirenti, M. The mathematical theory of dilute gases. Springer-Verlag, New York, 1994.

[7] Desvillettes, Some applications of the method of moments for the homogeneous Boltzmann equation. Arch. Rational Mech. Anal. 123 (1993), 387-395.

[8] Desvillettes, L., Mounot, C., About $L^{p}$ estimates for the spatially homogeneous Boltzmann equation. Ann. Inst. H. Poincaré Anal. Non Linéaire 22 (2005), 127-142.

[9] Desvillettes, L., Mounot, C., Large time behavior for the a priori bounds for the solutions to the spatially homogeneous Boltzmann equation with soft potentials. Preprint 2005.

[10] Desvillettes, L., Mounot, C. Entropy production estimates and asymptotic behavior for the spatially homogeneous Boltzmann equation without cutoff. Work in progress.

[11] Desvillettes, L., Wennberg, B. Smoothness of the solution of the spatially homogeneous Boltzmann equation without cutoff. Comm. Partial Differential Equations 29 (2004), 133-155. 
[12] Elmroth, T. Global boundedness of moments of solutions of the Boltzmann equation for forces of infinite range. Arch. Rational Mech. Anal. 82, 1 (1983), 1-12.

[13] Fournite, N. Uniqueness for a class of spatially homogeneous Boltzmann equations without cutoff for pseudo-Maxwellian molecules. Preprint n 12/2005 of Laboratoire d'Analyse et de Mathématiques Appliquées, Université Paris 12.

[14] Gustafsson, T. $L^{p}$-estimates for the nonlinear spatially homogeneous Boltzmann equation. Arch. Rational Mech. Anal. 92, 1 (1986), 23-57.

[15] Gustafsson, T. Global $L^{p}$-properties for the spatially homogeneous Boltzmann equation. Arch. Rational Mech. Anal. 103, 1 (1988), 1-38.

[16] Ikenberry, E., Truesdell, C. On the pressures and the flux of energy in a gas according to Maxwell's kinetic theory. I. J. Rat. Mech. Anal. 5 (1956), 1-54.

[17] Illner, R., Victory, H. D., Dukes, P., Bobylev, A. V. On Vlasov-Manev equations. II. Local existence and uniqueness. J. Statist. Phys. 91 3-4 (1998), 625-654.

[18] Lu, X., And Wennberg, B. Solutions with increasing energy for the spatially homogeneous Boltzmann equation. Nonlinear Anal. Real World Appl. 3 (2002), 243-258.

[19] Mischler, S., And Wennberg, B. On the spatially homogeneous Boltzmann equation. Ann. Inst. H. Poincaré Anal. Non Linéaire 16 (1999), 467-501.

[20] Mounot, C., Villani, C. Regularity theory for the spatially homogeneous Boltzmann equation with cut-off. Arch. Rational Mech. Anal. 173 (2004), 169-212.

[21] Моuнот, C. Quantitative lower bounds for the full Boltzmann equation, Part I: Periodic boundary conditions. Comm. Partial Differential Equations 30 (2005), 881-917.

[22] Toscani, G. and Villani, C. Sharp entropy dissipation bounds and explicit rate of trend to equilibrium for the spatially homogeneous Boltzmann equation. Comm. Math. Phys. 203 (1999), 667-706.

[23] Toscani, G. and Villani, C. On the trend to equilibrium for some dissipative systems with slowly increasing a priori bounds. J. Statist. Phys. 98 (2000), 1279-1309.

[24] Toscani, G. and Villani, C. Probability metrics and uniqueness of the solution to the Boltzmann equation for a Maxwell gas. J. Statist. Phys. 94 (1999), 619-637.

[25] Villani, C. Contribution à l'étude mathématique des équations de Boltzmann et de Landau en théorie cinétique des gaz et des plasmas. PhD thesis, Univ. Paris Dauphine, France, 1998.

[26] Villani, C. On a new class of weak solutions for the spatially homogeneous Boltzmann and Landau equations. Arch. Rational Mech. Anal. 143 (1998), 273-307.

[27] Villani, C. A review of mathematical topics in collisional kinetic theory. Handbook of mathematical fluid dynamics, Vol. I, 71-305, North-Holland, Amsterdam, 2002.

[28] Wennberg, B. On moments and uniqueness for solutions to the space homogeneous Boltzmann equation. Transport Theory Statist. Phys. 23 (1994), 533-539.

[29] Wennberg, B. Entropy dissipation and moment production for the Boltzmann equation. $J$. Statist. Phys. 86 (1997), 1053-1066.

[30] Wennberg, B. Regularity in the Boltzmann equation and the Radon transform. Comm. Partial Diff. Equations 19 (1994), 2057-2074. 